\title{
Entre pares soluciones integrales
}

\author{
María Clarissa Arenas Hinojosa*
}

\begin{abstract}
Resumen
El proyecto de vinculación Entre pares soluciones integrales (EPSI), ha logrado consolidar al Centro de Bachillerato Tecnológico Agropecuario de Hermosillo (CBTA) 264, como una unidad académica receptora. Primeramente, ha permitido que el perfil de egreso de las estudiantes de trabajo social desarrolle estrategias didácticas innovadoras que promuevan y accedan al análisis y la reflexión, a partir de experiencias vividas por los propios actores sociales de la comunidad estudiantil. En segundo, EPSI ha facilitado concretar y visibilizar el proyecto de vinculación y extensión universitaria firmada entre la Universidad de Sonora y el CBTA 264.

El curso taller se ha replicado en dos ocasiones y en ambos casos se retoman argumentos de convivencia, participación incluyente y habilidades para la vida; toda vez que se parte del concepto base de la educación como núcleo edificador de saberes y experiencias altamente significativas. EPSI está diseñado para reconocer los intereses de la comunidad educativa, incluyendo a los y las estudiantes, los docentes, los padres de familia, así como otros actores sociales involucrados e impactados por la labor académica. El objetivo final de EPSI es sumar esfuerzos para formar personas en la labor del autoconocimiento y reconocimiento de distintas vivencias y experiencias formativas: se fortalece así el trabajo colaborativo a fin de asumir una actitud de respeto e inclusión entre pares, docentes y comunidad.
\end{abstract}

\section{Introducción}

La población asentada en el poblado Miguel Alemán de la Costa de Hermosillo, ha interactuado a través de diferentes procesos, de igualdad desigualdad, abiertos encubiertos o cerrados, asumidos conscientes o enajenados, de conflicto; es decir, de ruptura o cooperación, competencia o marginación y en donde lo transversal es la unidad educativa: la escuela.

La escuela hoy por hoy sigue siendo el espacio en donde se busca privilegiar la integración social de las personas, legitimar el reconocimiento de los otros y las otras; es decir, sostener una convivencia sana, respetar la diversidad,

a la multiculturalidad, en general, a la dignidad del individuo, como atributo esencial de los derechos humanos.

\footnotetext{
${ }^{*}$ Maestra de tiempo completo de la Licenciatura en Trabajo Social, Universidad de Sonora. Correo: marenas@sociales.uson.mx
} 
Sin embargo, los espacios educativos constituyen una "fuente natural de conflictos, o en verdaderas oportunidades para aprender a vivir juntos en un espacio de paz" (Soriano, 2009).

La experiencia de la cual se da cuenta en las siguientes líneas, se desarrolla en una comunidad asentada en una zona agrícola fundada como comisaría en 1986, aunque sus orígenes datan de 1920 , cuando se asentaron los primeros grupos de agricultores y ganaderos.

De acuerdo con datos proporcionados por El Instituto Nacional de Estadística y Geografía (INEGI), en el poblado Miguel Alemán hay 30869 habitantes, 15995 hombres y 14874 mujeres. El ratio mujeres/hombres es de 0.930 , y el índice de fecundidad es de 2.77 hijos por mujer. Del total de la población, el $33.08 \%$ proviene de fuera del estado de Sonora. El $7.80 \%$ de la población es analfabeta (el $7.09 \%$ de los hombres y el $8.56 \%$ de las mujeres). El grado de escolaridad es de 5.98 ( 6 en hombres y 5.96 en mujeres).

El $12.20 \%$ de la población es indígena, y el $6.64 \%$ de los habitantes habla una lengua indígena. El $0.30 \%$ de la población habla una lengua indígena y no habla español.

El $38.87 \%$ de la población mayor de doce años está ocupada laboralmente (el $52.55 \%$ de los hombres y el $24.17 \%$ de las mujeres).

En Miguel Alemán hay 8150 viviendas. De ellas, el $95.46 \%$ cuentan con electricidad, el $97.41 \%$ tienen agua entubada, el $94.41 \%$ tiene excusado o sanitario, el $51.86 \%$ radio, el $87.64 \%$ televisión, el $74.25 \%$ refrigerador, el $42.69 \%$ lavadora, el $33.34 \%$ automóvil, el $11.27 \%$ una computadora personal, el $23.56 \%$ teléfono fijo, el $65.68 \%$ teléfono celular, y el $5.63 \%$ internet.

De acuerdo con el grado de nivel patrimonial definido por la Asociación
Mexicana de Agencias de Investigación de Mercado (AMAI) un $56 \%$ de estos hogares se ubica en el nivel $D$, en donde el jefe de hogar tiene estudios hasta primaria. Solamente el $4 \%$ cuenta con conexión a internet fijo en la vivienda. Un poco menos de la mitad de su gasto (46 \%) se destina a la alimentación.

En este contexto, el Centro de Bachillerato Tecnológico Agropecuario (CBTA) 264, atiende año con año un promedio de 748 estudiantes, de los cuales 368 son hombres, mientras que 380 son mujeres. Estos jóvenes, en algunos casos, asumen conductas violentas, uso de substancias adictivas, y en otros casos problemas relacionados a la salud mental y reproductiva, debido a su condición de vulnerabilidad y manejo de competencias relacionales asociadas al hecho de haber sufrido castigos físicos durante la infancia, falta de afecto, y de vínculos emocionales, pertenencia a una familia disfuncional y haber presenciado en repetidas ocasiones conflictos conyugales.

Contextualización y reconstrucción de la práctica

La sociedad es un collectivum, y como tal, algo más que la simple suma de individuos o de voluntades. En nuestro caso, la población objetivo está constituida por una comunidad estudiantil cuyas familias provienen de distintas localidades del estado y de la república, cuyas etnias hablan distintas lenguas, así como de residentes de la localidad del poblado Miguel Alemán a quienes les distinguen historias asimétricas. Ambos grupos poblacionales han interactuado a través de diferentes procesos, de igualdad desigualdad, abiertos encubiertos o cerrados, asumidos conscientes o enajenados, de conflicto; es decir, de ruptura o cooperación, competencia o marginación y en donde lo 
transversal es la unidad educativa: la escuela.

La escuela hoy por hoy sigue siendo el espacio en donde se busca privilegiar la integración social de las personas, legitimar el reconocimiento de los otros y las otras; es decir, sostener una convivencia sana, respetar la diversidad, a la multiculturalidad, en general, a la dignidad del individuo, como atributo esencial de los derechos humanos.

Sin embargo, los espacios educativos constituyen una "fuente natural de conflictos, o en verdaderas oportunidades para aprender a vivir juntos en un espacio de paz" (Soriano Díaz, 2009).

Para mediadores como Folger y Jones (1997), la gestión del conflicto comienza:

Rechazando la premisa básica de que los conflictos deben verse como problemas. Las disputas que surgen de las preocupaciones, las insatisfacciones, las tensiones interpersonales o relacionales sustantivas pueden verse no como problemas sino como oportunidades para el crecimiento y la transformación humana. Específicamente, en esta orientación transformadora el conflicto es visto como una ocasión potencial para el crecimiento en dos dimensiones críticas del desarrollo humano: 'la adquisición de poder' y el 'reconocimiento'.

El CBTA 264, atendía para el semestre 2016-2 a una población de 396 hombres y 438 mujeres. Para el 2017-2 recibía aproximadamente a 630 estudiantes más, según datos proporcionados por la directora del plantel, doctora Dalia Ivvon Castro Quiroz, quien resalta que se han identificado en un alto porcentaje, problemas en los temas de salud, violencia, conductas vandálicas, reproductiva, adicciones, entre otros.

Nos encontramos con realidades que duelen, con estadísticas más hacia la intolerancia y la marginación. Por ello, es necesario reforzar las habilidades personales de los propios actores sociales inmersos en la comunidad estudiantil, para saber relacionarse pacíficamente, para demostrar actitudes positivas hacia la convivencia pacífica o de implicarse en la comunidad, para promover en ellas relaciones positivas entre personas y grupos con intereses y percepciones afines $o$ en su caso opuesto.

El sentido de presentar un proyecto de intervención a través del curso taller Entre pares soluciones integrales (EPSI), en donde se retoman argumentos de convivencia relacional, participación incluyente y habilidades para la vida, parte de la necesidad de ver a la educación como la construcción de saberes y experiencias lo más altamente significativas. Está basado en el reconocimiento de los intereses de la comunidad educativa: estudiantes, docentes, padres de familia y todos los demás actores sociales involucrados. Todo ello, con el objeto de sumar esfuerzos para formar personas en el autoconocimiento y reconocimiento de las diferencias, de vivencias y experiencias de vida, apoyado en el trabajo colaborativo, a fin de asumir una actitud de respeto e inclusión entre pares, docentes y comunidad.

Finalmente se subraya el hecho de que este proyecto se enmarca en el actual Plan de Desarrollo Institucional (20132017) en tanto que dirige su intervención hacia la población en estado de vulnerabilidad, como se indicó anteriormente, reafirmando la misión y visión de la Universidad de Sonora a través de acciones de vinculación y extensión universitaria (el Plan de Desarrollo Departamental, 2013- 2017, en el punto 5 en lo referente a incrementar la vinculación con las necesidades de los sectores público, social y privado del 
estado), que permitan a estudiantes fortalecer su perfil de egreso, desempeñando funciones de acuerdo con su campo disciplinar con la integración de actividades desde lo local, con una intervención multidimensional.

\section{Objetivos}

Promover la interacción de líderes pares de la comunidad estudiantil del CBTA 264 a través de la implementación del curso taller EPSI, a fin de desarrollar competencias individuales y grupales en la participación incluyente y la convivencia basada en el respeto de sí mismo y de los demás, en el marco de la convivencia para la paz.

\section{Específicos}

$>$ Fomentar la dimensión ética y trascendente de la persona.

$>$ Desarrollar un pensamiento creativo a través de la reflexión y el debate.

$>$ Fortalecer entre los estudiantes los potenciales ético afectivo, creativo y comunicativo.

$>$ Consolidar su identidad, la aceptación de sí mismos, que desarrollen una autoestima saludable, facilitando la toma de decisiones lo más asertivo posible para su proyecto de vida.

$>$ Promover el desempeño de competencias disciplinares del trabajo social en el ejercicio del curso taller EPSI.

El diseño de la planeación del curso taller, fue a través de cinco módulos secuenciados, diferenciados y coherentes con los objetivos y metas propuestas. Se llevó a cabo durante el periodo de septiembre a noviembre del 2016, con un promedio de dos horas cada semana mes. Cabe señalar sobre todo, al elemento de la flexibilidad en cuanto a márgenes de extensión que permitieron afrontar situaciones imprevistas, como cancelación de sesiones derivadas por factores tales como fechas cívicas y/o actividades propias del plantel que involucraban a todos los estudiantes del CBTA 264. Dicho lo anterior, el trabajo desarrollado consistió en la realización de cinco módulos:

\section{Módulo I: introducción a la mediación}

Objetivo: introducir al blindaje de la mediación como herramienta de gestión de conflictos en la construcción de un modelo de convivencia pacífica.

$>$ Importancia de la mediación en el ámbito escolar

$>$ Voluntariedad,

> Participación activa de las partes

$>$ Flexibilidad

$>$ Confidencialidad

$>$ Neutralidad

\section{Módulo II: potencial afectivo}

Objetivo: fortalecer conceptual y metodológicamente el potencial afectivo, como un primer paso en el proceso de configuraciones de subjetividades políticas orientadas a la construcción de la paz.

> Potenciación de la subjetividad (autorreconocimiento)

$>$ Identificación de sentimientos

$>$ Potenciación de intersubjetividad

> Potenciación de la ciudadanía y la acción afectiva

\section{Módulo III: potencial ético}

Objetivo: brindar herramientas conceptuales y metodológicas sobre ética, a fin de que participen en la transformación pacífica de conflictos.

$>$ Perspectivas éticas en la construcción de la paz

$>$ Capacidades funcionales humanas centrales 


\section{Módulo IV: potencial creativo}

Objetivo: proporcionar herramientas conceptuales de la transformación del conflicto, haciendo uso de la mediación.

$>$ Conflictos

$>$ La mediación como herramienta de transformación de conflictos

\section{Módulo V: potencial comunicativo}

Objetivo: brindar herramientas conceptuales y metodológicas que permitan establecer una comunicación asertiva con el otro en los procesos de construcción de la paz.

> Barreras de la comunicación

$>$ Comunicación asertiva

$>$ Análisis crítico de los medios de comunicación

$>$ Comunicación

$>$ alternativa

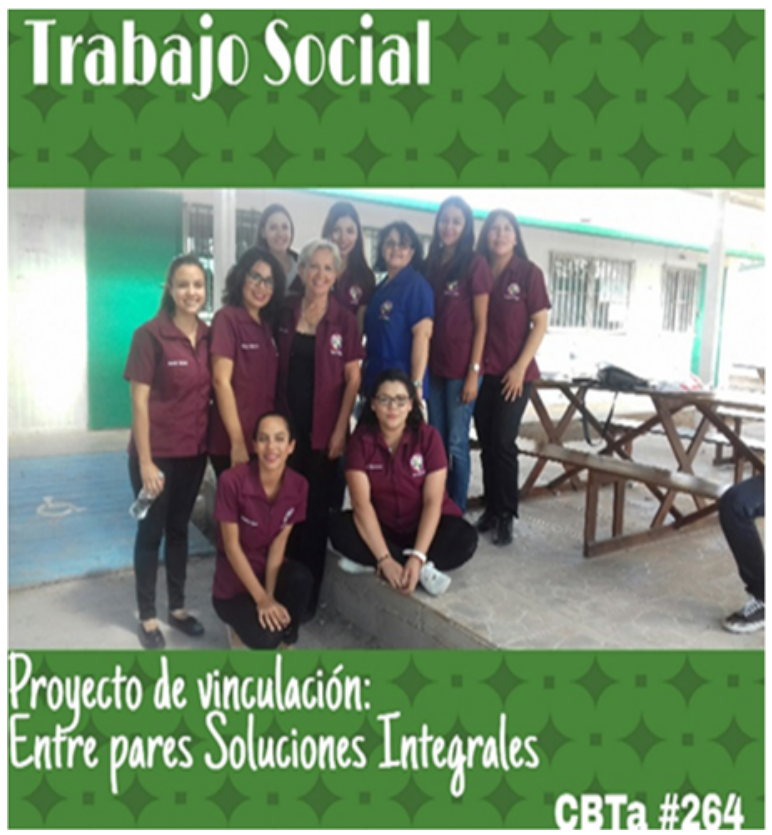

Foto 1: María Clarissa Arenas Hinojosa (2016). Equipo de trabajo. Universidad de Sonora.
Descripción de la práctica

Se parte de la premisa de que la práctica escolar (PE) se concibe en un doble sentido: como práctica de enseñanza, propia de cualquier proceso formativo; y como apropiación del oficio de la profesión, cómo iniciarse, perfeccionarse y/o actualizarse. La unidad educativa del CBTA 264, se integra a los espacios educativos que buscan reproducir de manera escalonada el proceso metodológico del trabajo social.

Por tanto, los estudiantes del CBTA 264 , se suman al proyecto en primera instancia, a través de un proceso de convocatoria y de sensibilización en la comunidad estudiantil de dicho centro, procediendo en la identificación de estudiantes, cuyo perfil embone en la categoría de líder. Con ello se integra el grupo de pares, con veintisiete jóvenes, de los cuales dieciocho son mujeres y nueve, varones; es decir, que en este segmento de la población estudiantil, el $66,67 \%$ predomina el género femenino. En cuanto a la edad, el 33,33\% asegura contar con diecisiete años de edad, mientras que el $7,41 \%$, afirma tener catorce años cumplidos; en cuanto al lugar de nacimiento que ocupan en su familia, el $40,74 \%$ es decir, once de los veintisiete jóvenes, representa el primer nacimiento en sus respectivas familias, y solamente dos jóvenes con el 7,41\% nacieron en el sexto lugar. Sus familias de origen en un $78 \%$ migraron del interior del país, particularmente de los estados de Chiapas, Oaxaca, Veracruz, Guerrero y Sinaloa; mientras que el $22 \%$ provienen de diferentes municipios de Sonora.

Para explorar un poco sobre la autoestima de los integrantes de este grupo, se les aplicó un cuestionario de autoestima de Rosemberg. Esta escala de medición es entendida como los sentimientos de valía personal y de respeto a sí mismo, incluye diez ítems con 
frases de las que cinco están enunciados de forma positiva y el resto, de forma negativa. Se logró identificar que el $15 \%$ de los participantes refleja una autoestima elevada, considerada como autoestima normal; el $55 \%$ como autoestima baja, que de acuerdo con esta escala, existen problemas significativos de autoestima; y el $30 \%$ presenta una medición de autoestima media, la cual no presenta problemas de autoestima graves, pero es conveniente mejorarla, lo que confirma la necesaria intervención del profesional de trabajo social para coadyuvar en el fortalecimiento académico, personal y de desarrollo humano de estos jóvenes.

En el curso taller se utilizaron técnicas didácticas como las expositivas y audiovisuales que giraron en torno a generar ambientes de trabajo colaborativos, de participación activa, de integración y de reflexión. En los temas abordados por los y las practicantes, se consideró la incorporación del análisis temático, la mayor aproximación a situaciones problemáticas vividas en los entornos áulicos, con objeto de mejorar la convivencia entre pares y favorecer el adecuado cumplimiento de los objetivos educativos. Todo esto, en un clima que propiciara el desarrollo integral de los estudiantes por medio del aprendizaje basado en incidentes críticos $A B I C$; toda vez que esta técnica ofrece una fuente de información que visibiliza los problemas de gestión de aula relacionados con la convivencia. Lo anterior, primero para entender los incidentes críticos ocurridos; $y$ en segundo momento, el incidente crítico, al convertirse en una oportunidad de reflexión, pone en marcha mecanismos de autorregulación, generando nuevo conocimiento $y$ recursos tanto cognitivos, como emocionales y comunicacionales (Nail Kröyer, Gajardo Aguayo y Muñoz Reyes, 2012).
Interpretación crítica

Diez y Gachi (1999), citan en su texto Herramientas para trabajar en mediación: "El mediador es un curioso que, pendido de las partes, se mete dentro de las conversaciones sobre el problema para ir "destapando" algún viejo canal de comunicación o para ayudarlos a construir puentes que los acerquen a algún territorio común".

Dentro de ese perfil de curiosidad, las estudiantes de la Licenciatura en Trabajo Social buscaron, por medio de actividades lúdicas, colocar en la mesa del análisis, situaciones de incidentes críticos (ABIC), las formas en que a juicio de los propios involucrados receptores del curso taller EPSI, dieron solución a estos $y$, en prospectiva cuáles serían los cambios a introducir a nivel de razonamiento, emoción e imaginación.

Es decir, que a diferencia de un trabajo totalmente expositivo, en donde los involucrados estuviesen como simples receptores, se buscó colocar las condiciones pertinentes para analizar, reflexionar, comunicar y socializar aquellos problemas más recurrentes en su entorno socioeducativo familiar. Las experiencias dieron como resultado positivo el involucramiento cada vez mayor de los jóvenes en plantear conflictos y formas de resolverlos en un ambiente de paz.

Mientras que en las primeras sesiones de trabajo se tenía que recurrir al llamado de los líderes, conforme se fue avanzando en la agenda, los jóvenes, prestos a recibirnos en el aula dispuesta para los trabajos áulicos, aguardaban nuestra llegada. Es decir, que con esa actitud se confirmó la hipótesis de que los jóvenes aceptaron el rol, y encontraron en ese espacio del curso taller EPSI, un lugar de respeto y solidaridad. 
Cabe mencionar que previo a cada reunión y de acuerdo con el módulo y/o tema, las estudiantes de trabajo social analizaban la dinámica grupal, identificando los distintos roles que al interior del grupo se asumían; así mismo, hacían registro en cuaderno de notas, así como del gráfico de cada sesión de trabajo. Es decir, que la instancia educativa del CBTA 264, fungió como unidad receptora permitiendo que el perfil de egreso de las estudiantes del noveno semestre, adquiriera un mayor nivel de desempeño al desarrollar estrategias didácticas innovadoras, que permitieran el análisis y la reflexión a partir de experiencias vividas por los propios actores sociales de la comunidad estudiantil.

Enseguida se muestra un gráfico elaborado a partir de una de las sesiones en donde se analiza bajo la mirada del trabajo social, cómo se interrelacionaban los jóvenes, si hubo o no modificación entre las alianzas internas, o si surgían otras rupturas en general, sobre la dinámica del grupo.

\section{Representación gráfica de la estructura del grupo}

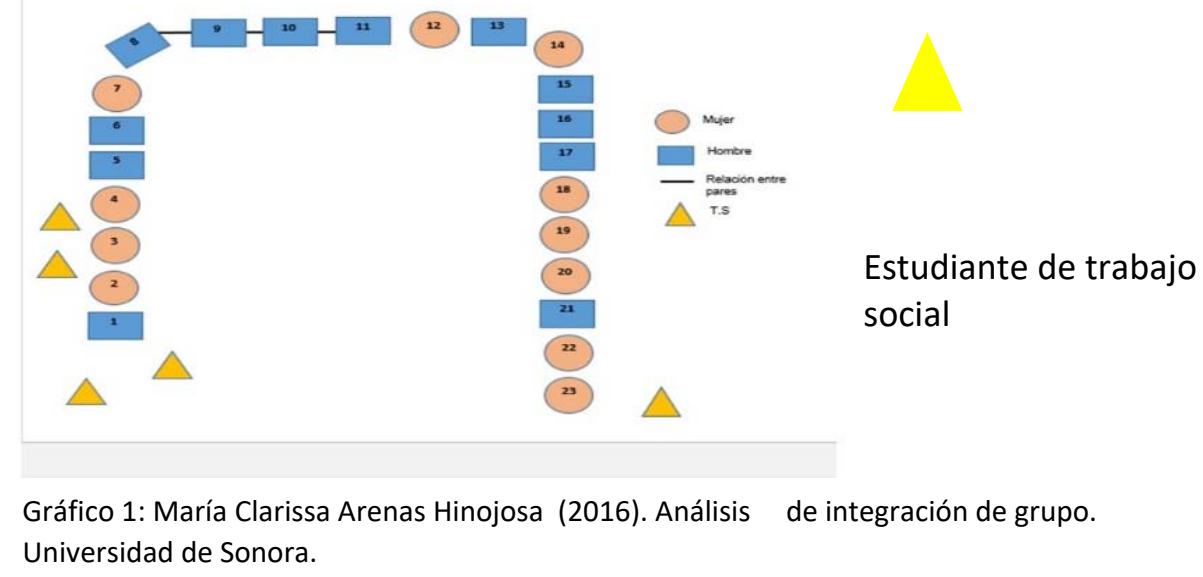

En el gráfico 1, durante la primera sesión, se aprecia la distribución de los jóvenes y cómo en el fondo de la herradura, la relación entre cuatro de ellos fue estrecha.

En cada sesión, además de atender lo propio de lo programado y de acuerdo con el clima vivenciado, los mismos jóvenes fueron incorporando códigos de ética tales como el saludo de mano al despedirse de las sesiones; más adelante fue el abrazo de grupo, el escuchar con atención y participar de manera activa, pero a través del trabajo colaborativo, respetando género, edad y liderazgo. Esto propició que aquellas voces silenciadas por los mismos compañeros, se pronunciaran de forma simétrica, es decir, en un ambiente de paz.

Los principales problemas que acusan los jóvenes estudiantes del CBTA 264 son: el de dar una pronta atención al problema del suministro indiscriminado de sustancias al interior del plantel, ya que esto genera violencia dentro y fuera del mismo. 


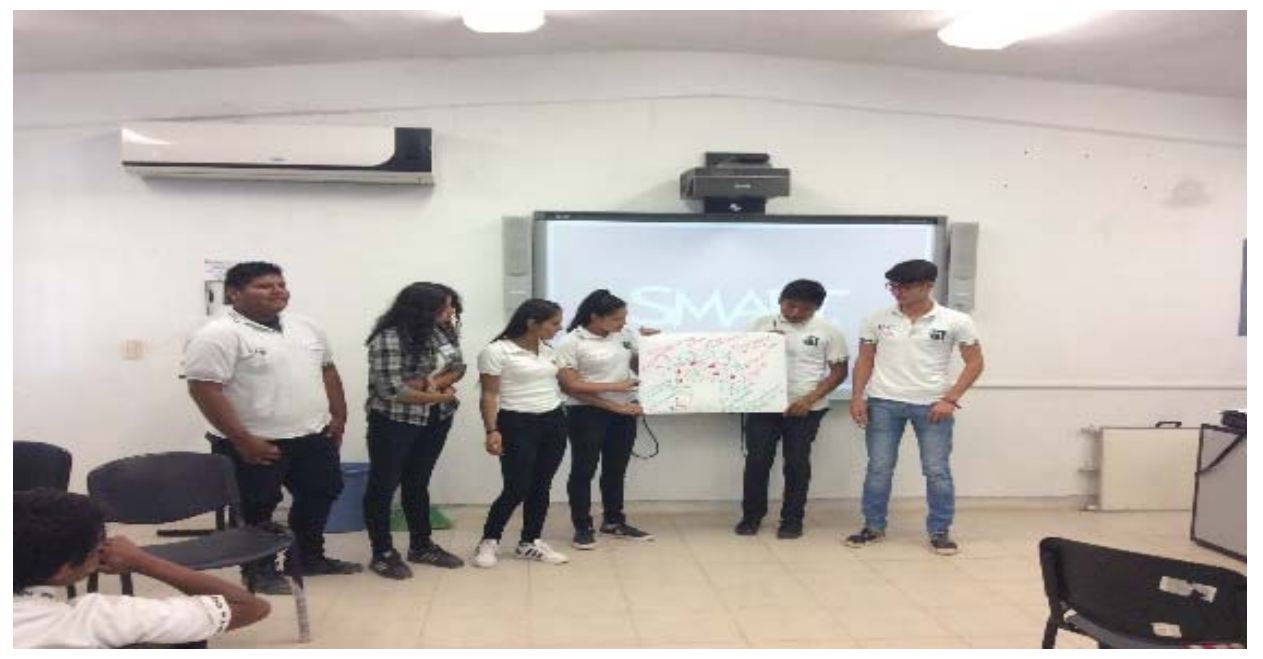

Foto 2: María Clarissa Arenas Hinojosa (2016). Exposición árbol de problemas. Universidad de Sonora.

Lo anterior fue doblemente ratificado en la evaluación, ya que se les solicitó que construyeran en equipo un árbol de problemas; así mismo, la lluvia de ideas generó elementos de discusión y de análisis concluyendo con una magnífica reflexión del entorno que les rodea; también lo referente a la cuestión social tan marcada en lo que tiene que ver con procesos de ruptura, exclusión, marginación, que sumados a la situación de precariedad y la embestida de grupos del crimen organizado, violentan la paz y la seguridad de la población en general. Por lo que a juicio de los propios actores sociales, en este caso de los jóvenes de bachillerato, se requiere fortalecer la cultura de la paz, de respeto y de igualdad. Los jóvenes valoraron en los potenciales ético, afectivo, creativo y comunicativo, la posibilidad de trabajar al unísono en su comunidad para consolidar su propia identidad, contribuir en la construcción de un tejido social inclusivo, de respeto y solidaridad.

\section{Conclusiones}

a) $\mathrm{Si}$ bien es cierto, el proyecto de vinculación EPSI, en la vía de los hechos surge a partir de la iniciativa de la academia de dar pronta respuesta a un reclamo social. Este proyecto propició un espacio de interacción tanto de los jóvenes receptores del CBTA 264, como de los practicantes de trabajo social. Se colocó como centro de atención, la reflexión y análisis de las voces silenciadas por la opacidad de los propios actores sociales involucrados en la unidad receptora que, al tomar notoriedad, encontraron un reflejo y eco en sus demandas personales y colectivas.

b) EPSI fortalece el perfil de egreso de los practicantes de trabajo social al incorporarlos en su formación, la preparación en la mediación y en constructores de paz. 
c) El CBTA 264, ubicado en el Poblado Miguel Alemán en la Costa de Hermosillo, en la actualidad cuenta con un poco más de setecientos jóvenes. Dichos estudiantes están expuestos a la presión de pares, de un medio social con reducidos espacios culturales y de esparcimiento, sumado a ello, la presencia del crimen organizado y pandillas. Por lo anterior, es una unidad receptora que brinda la oportunidad para incorporar, no solamente la mirada del trabajo social, sino acciones multidisciplinares cuyo objetivo sea el promover actividades colectivas que alcancen un nivel mayor de participación ciudadana, de inclusión y de colaboración.

\section{Prospectiva}

En nuestro caso, la finalidad de la prospectiva no es adivinar el futuro, sino trabajar en ello. Es por ello que entender el ahora es repensar en lo que está pasando, ¿cuál es su etiología?, ¿por qué sucede?, ¿qué queremos?, ¿qué rumbo llevamos o hacia dónde vamos?

Precisamente al dar respuesta a esos cuestionamientos es como se logra ubicar en el plano de la construcción de escenarios futuros, para que desde esa plataforma se diseñe y proyecte nuestras acciones en el presente.

En nuestro caso particular, la réplica del proyecto de vinculación EPSI, se explica como una opción que requiere indiscutiblemente algunas adecuaciones en términos de incorporar, de forma paralela, grupo de docentes y de padres de familia, a fin de integrar todos los subsistemas inmersos en el sistema educativo.

También, ampliar acciones que promuevan la creatividad entre los jóvenes de bachillerato, a través de convocatorias de elaboración de carteles, campañas de construcción de paz, preventivos en el tema de elección de decisiones, manejo del conflicto, entre otros. Además, trasladar la expo feria universitaria para que los residentes ubiquen áreas de oportunidad y continúen con su proyecto de vida.

\section{Bibliografía}

Centro de la Investigación Educativa y Capacitación Institucional, S.C. (CIECI). (2016). Diseño y evaluación de situaciones didácticas, curso impartido por Patricia Frola. Universidad de Sonora.

Diez, F. y Tapia, G. (1999). Herramientas para trabajar en mediación. Buenos Aires: Paidós.

Folger, J.P. y Jones, T.S. (1997). Nuevas direcciones en mediación: investigación y perspectivas comunicacionales. Argentina: Paidós.

Grover Duffy, K., Grosch, J.W. y Olczak, P.V. (1996). La mediación y sus contextos de aplicación. Barcelona: Paidós.

Matilla Castellanos, L., Bravo, A. y Martínez Ruiz, V. (2003). Habilidades para la vida, una propuesta educativa para convivir mejor. Bogotá: $\mathrm{Fe}$ y Alegría.

Ministerio de Educación Nacional. (2011). Orientaciones para la institucionalización de las competencias ciudadanas. Cartilla 1. Brújula. Programa de Competencias Ciudadanas. Bogotá: Amado Impresores S.A.S. Recuperado de: http://www.mineducacion.gov.co/1759/a rticles-235147_archivo_pdf_cartilla1.pdf 
Nail Kröyer, O., Gajardo Aguayo, J. y Muñoz Reyes, M. (2012). La técnica de análisis de incidentes críticos: Una herramienta para la reflexión sobre prácticas docentes en convivencia escolar. Psicoperspectivas, 11(2), 5676.

Rosenberg, M. (1965). Atienza, F.L., Balaguer, I. y Moreno, Y. (2000). Escala de autoestima de Rosenberg.

Recuperado de http:// www.uv.es/ uipd/ cuestionarios/accesolibre/EAR.pdf

Salazar Castilla, M. y Loaiza de la Pava, J.A. (2014). Procesos de Construcción de Paz y Democracia con Participación de Niños, Niñas y Jóvenes. Centro de Estudios Avanzados en Niñez y Juventud de la Universidad de Manizales, Colombia. Diplomado internacional celebrado en la Universidad de Sonora.

Soriano Díaz, A. (2009). Violencia y conflicto. La escuela como espacio de paz. Profesorado Revista de curriculum y formación del profesorado, 13(1)321334. Recuperado de file:///C:/Users/ Estilo/Downloads/42172-129915-1-SM. pdf
Suares, M. (1996). Mediación. Conducción de disputas, comunicación y técnicas. Buenos Aires: Paidós.

UNESCO. (2012). Educación para la convivencia y la cultura de paz en América Latina y el Caribe. Recuperado el 10 de febrero de 2015 de http://www.unesco.org/new/fileadmin/M ULTIMEDIA/FIELD/Santiago/pdf/Educa cion-Convivencia-Paz.pdf

\section{Ilustraciones}

Arenas Hinojosa, M.C. (2016). Equipo de Trabajo. [Fotografía]. Universidad de Sonora.

Arenas Hinojosa, M.C. (2016). Análisis de integración de grupo. [Gráfico1]. Universidad de Sonora.

Arenas Hinojosa, M.C. (2016). Exposición árbol de problemas. [Fotografía]. Universidad de Sonora.

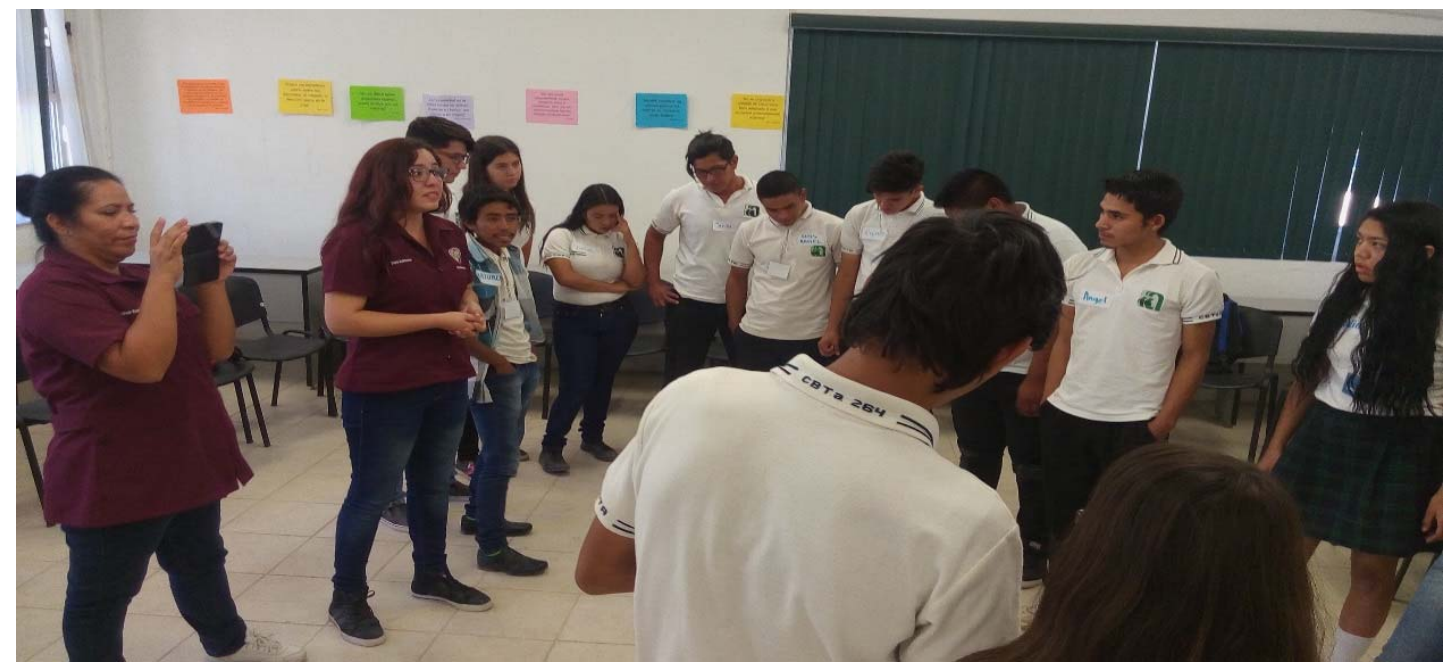

Foto 3: María Clarissa Arenas Hinojosa (2016). Sesión de Trabajo. Universidad de Sonora. 